\title{
Development of chimeric molecules that degrade the estrogen receptor using decoy oligonucleotide ligands
}

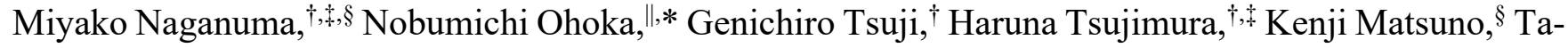 \\ kao Inoue," Mikihiko Naito, ${ }^{* *}$ and Yosuke Demizu ${ }^{\dagger,}, *$ \\ 'Division of Organic Chemistry, National Institute of Health Sciences, Kanagawa, Japan \\ ${ }^{\ddagger}$ Graduate School of Medical Life Science, Yokohama City University, Kanagawa, Japan \\ $\S^{\S}$ Department of Chemistry and Life Science, Kogakuin University, Tokyo, Japan \\ "Division of Molecular Target and Gene Therapy Products, National Institute of Health Sciences, Kanagawa, Japan \\ **Laboratory of Targeted Protein Degradation, Graduate School of Pharmaceutical Sciences, The University of Tokyo, Tokyo, \\ Japan
}

KEYWORDS: ubiquitin-proteasome system; protein knockdown; decoy; transcription factors; estrogen receptor

\begin{abstract}
Targeted protein degradation using chimeric small molecules, such as proteolysis-targeting chimeras (PROTACs) and specific and nongenetic inhibitors of apoptosis protein [IAP]-dependent protein erasers (SNIPERs), has attracted attention as a method to degrade intracellular target proteins via the ubiquitin-proteasome system (UPS). These chimeric molecules target a variety of proteins using small molecules that can bind to the proteins. However, it is difficult to develop such degraders in the absence of suitable small molecule ligands for the target proteins, such as for transcription factors (TFs). Therefore, we constructed the chimeric molecule LCL-ER(dec), which consists of a decoy oligonucleotide that can bind to the estrogen receptor $\alpha(\mathrm{ER} \alpha)$ and an IAP ligand, LCL161 (LCL), in a click reaction. LCL-ER(dec) was found to selectively degrade ER $\alpha$ via the UPS. These findings will be applicable to the development of other oligonucleotide-type degraders that target different TFs.
\end{abstract}

The identity of many target proteins that are involved in the pathogenesis of various diseases has been elucidated, and various drug discovery techniques have been developed to target these proteins. ${ }^{1,2}$ In particular, the use of targeted protein degradation has recently been attracting attention. This technology uses chimeric molecules called proteolysis-targeting chimeras (PROTACs) or specific and nongenetic inhibitors of apoptosis protein [IAP]-dependent protein erasers (SNIPERs) to degrade target proteins in cells via the ubiquitin-proteasome system (UPS). ${ }^{3,4}$ These chimeric molecules consist of a ligand for the target protein (protein of interest, POI) and an E3 ligand. The proximity of the POI and E3 ligase acts as a molecular mediator, thereby promoting the ubiquitination and subsequent degradation of the POI. ${ }^{2}$ Small molecules, such as LCL161 (LCL) for IAPs, VH032 (VH) for von Hippel-Lindau protein (VHL), and pomalidomide (POM) for cereblon (CRBN) are often used as ligands for E3 ligases. ${ }^{3}$ A small molecule that is known to bind to a POI can be used to develop chimeric molecules, and many chimeric degraders using small molecules as POI ligands have been developed to date. ${ }^{3}$ Because the only activity required for the ligand is for it to be able to bind to the POI, this technology can target proteins that have no enzymatic activity (i.e., for which inhibitors cannot be developed). However, it is difficult to develop small molecule-based degraders against a POI in the absence of appropriate ligands. An alternative approach is to use molecules, such as peptides, that can bind to the protein surfaces as POI ligands. Several groups have recently reported peptide-based degraders that target

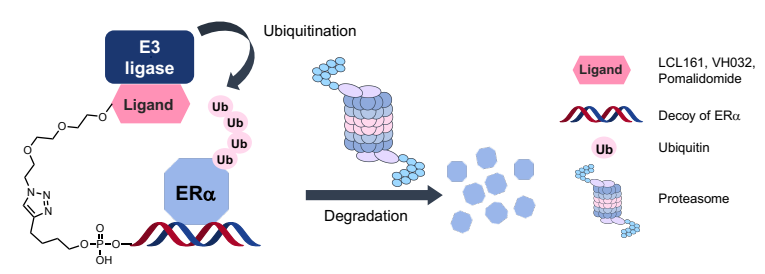

Figure 1. Decoy-type PROTACs degrade the ER $\alpha$ via the UPS.

transcriptional factors (TFs), such as the estrogen receptor $\alpha$ $(\mathrm{ER} \alpha)^{5}$ and neurogenic locus notch homolog protein 1 (NOTCH1), ${ }^{6}$ by introducing an oligopeptide that interacts with the surface of the POI. This peptide-based protein knockdown strategy is also applicable to several types of POIs that have no specific small-molecule ligands.

Other molecules that can bind to protein surfaces include oligonucleotides, such as aptamers ${ }^{7}$ and decoys ${ }^{8}$. If such oligonucleotides can be used as POI ligands in the development of chimeric degraders, the number of target proteins that can be degraded would be expanded. It is particularly advantageous to use a decoy as the ligand when targeting TFs, because the decoy can use the known DNA-binding sequence of the target TF, making it easy to design the ligand. In the present study, we developed decoy-based chimeric degraders that target the ER $\alpha$ 
5'-GTCAGGTCACAGTGACCTGAT-3' 3'-CAGTCCAGTGTCACTGGACTA-5' ER(dec)
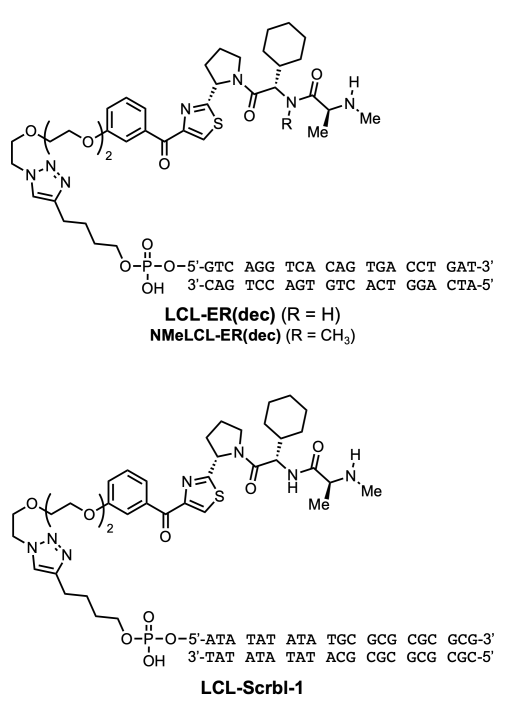
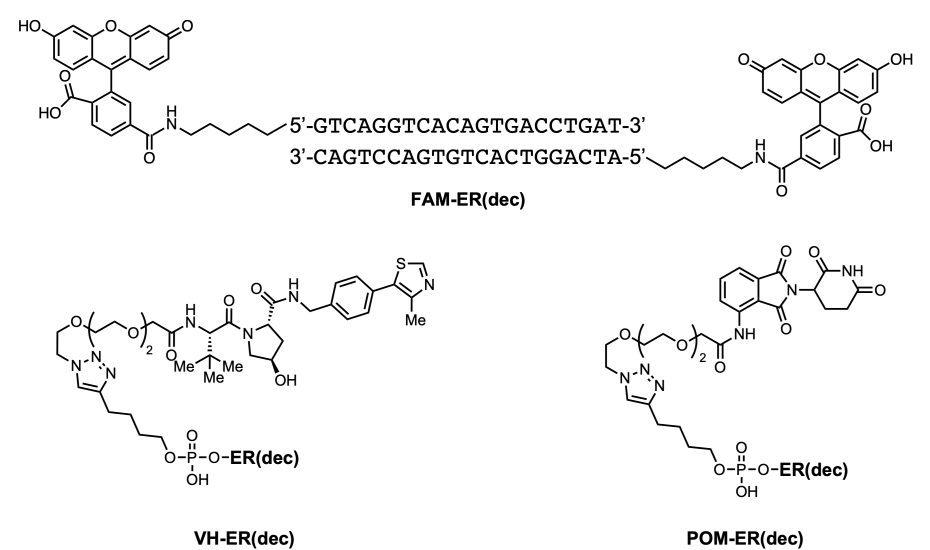

POM-ER(dec)
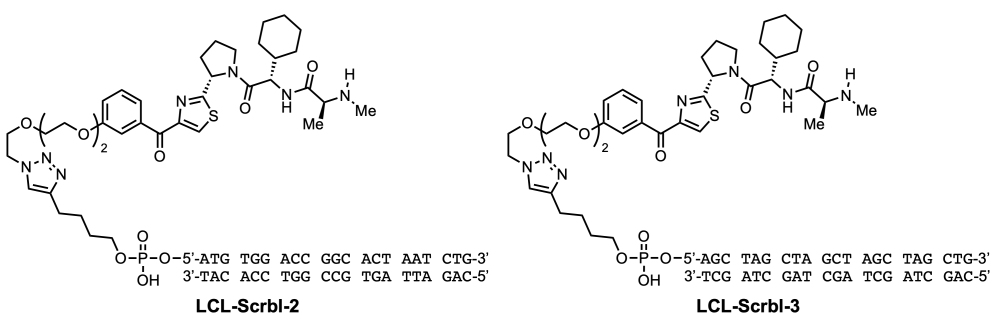

Figure 2. Molecules synthesized in this study.

as a model TF (Figure 1). The ER $\alpha$ is a member of the nuclear hormone receptor superfamily ${ }^{9}$ and can bind directly to DNA to act as a TF ${ }^{10}$ In addition, ER $\alpha$ homodimers can form transcriptional complexes on the DNA response sequence and regulate the expression of target genes by recruiting co-regulators. ${ }^{10} \mathrm{We}$ have previously developed small molecule-based chimeric degraders against $\mathrm{ER} \alpha^{11,12}$ using the ER $\alpha$ antagonist 4-hydroxytamoxifen (4-OHT), and peptide-based ER $\alpha$ degraders $^{5}$ using a coactivator motif that interacts with the ER $\alpha$ surface. Therefore, we chose ER $\alpha$, as a model TF, as the target for the development of oligonucleotide-type chimeric degraders. Specifically, we designed a thermodynamically stable double-stranded decoy, ER(dec), based on the sequence of the estrogen-responsive element $(\mathrm{ERE})^{13}$ that is known to bind strongly to the ER $\alpha$. This decoy was conjugated with three different E3 ligase ligands, LCL, VH, and POM, to construct the degraders LCL-ER(dec), VH-ER(dec), and POM-ER(dec), respectively. The structural properties, binding affinities, and ER $\alpha$-degradation activities of these degraders were evaluated.

The ER $\alpha$-binding decoy ER(dec) (21 residues) was designed using information from the DNA sequence and co-crystal X-ray structure of the ER $\alpha,{ }^{14}$ and an ERE from the Xenopus vitellogenin A2 gene. $^{13}$ The 5'-end of the sense strand 5'GTCAGGTCACAGTGACCTGAT-3' of ER(dec) was modified with a hexynyl group. To construct the E3 ligase ligands, a PEG3 linker with an azide group was attached to $\mathrm{LCL}, \mathrm{VH}^{15}$, and $\mathrm{POM}^{16}$. Then, the above sense strand with the alkyne was conjugated to each azide-containing E3 ligase ligand by a copper-catalyzed click reaction. Subsequent hybridization with the antisense strand 5'-ATCAGGTCACTGTGACCTGAC-3' afforded the desired chimeric molecules LCL-ER(dec), VHER(dec), and POM-ER(dec). In addition, three ER $\alpha$ non-binding decoys were designed with the same base composition as ER(dec): Scrbl-1 with an AT repeat and GC repeat sequence; Scrbl-2 with a completely randomized sequence; and Scrbl-3 with an AGCT repeat sequence. The chimeric molecules LCLScrbl-1, LCL-Scrbl-2, and LCL-Scrbl-3 were synthesized in a similar manner as LCL-ER(dec). A non-degradable control NMeLCL-ER(dec) containing an $N$-methylated analog of LCL161, and the fluorescein (FAM)-labeled decoy FAM$\mathbf{E R}(\mathbf{d e c})$ for use in a competitive fluorescence polarization assay were also synthesized (Figure 2). The detailed synthetic protocols of these molecules are described in the supporting information.

The preferred higher-order structures of the synthesized ER(dec) and the chimeric molecules LCL-ER(dec), VHER(dec), and POM-ER(dec) were analyzed using CD spectra. Each decoy showed a negative maximum at approximately 240 $\mathrm{nm}$ and a positive maximum at approximately $280 \mathrm{~nm}$, indicating that LCL-ER(dec), VH-ER(dec), and POM-ER(dec) formed typical right-handed B-type structures and the terminal modification of ER(dec) did not affect the higher-order structure (Figure S4). The melting temperature $(\mathrm{Tm})$ values of ER(dec), LCL-ER(dec), VH-ER(dec), and POM-ER(dec) were $64.5,64.3,66.9$, and $62.9{ }^{\circ} \mathrm{C}$, respectively, which indicated that these molecules had similar conformational stability to $\mathbf{E R ( d e c ) ~ ( F i g u r e ~ S 6 ) . ~}$

The binding affinities of ER(dec), LCL-ER(dec), VHER(dec), and POM-ER(dec) to the ER $\alpha$ were evaluated by a competitive fluorescence polarization assay. For the evaluation, the compounds were added to a buffer system containing ER $\alpha$ and FAM-ER(dec), and the inhibitory concentration was calculated as the $\mathrm{IC}_{50}$ value. The $\mathrm{IC}_{50}$ values were $83.2 \mathrm{nM}$ for ER(dec), $37.9 \mathrm{nM}$ for LCL-ER(dec), $39.3 \mathrm{nM}$ for VH- 
A

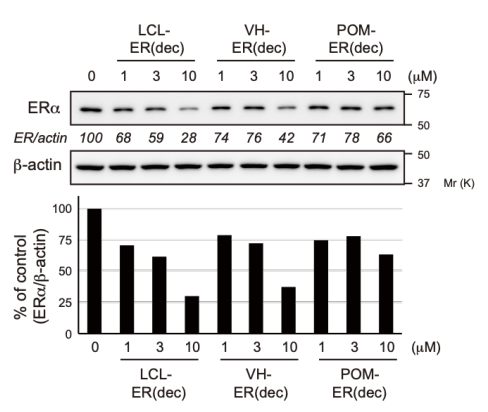

D

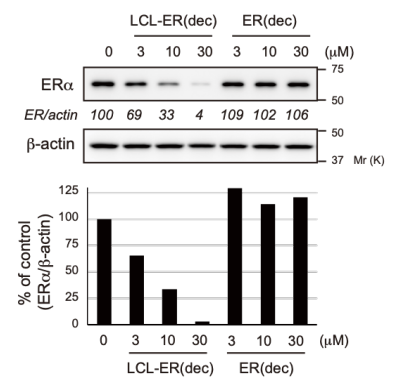

B

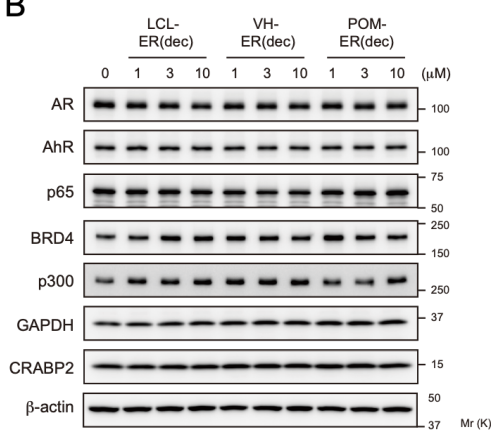

$\mathrm{E}$

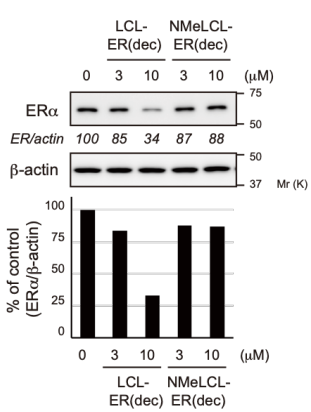

C

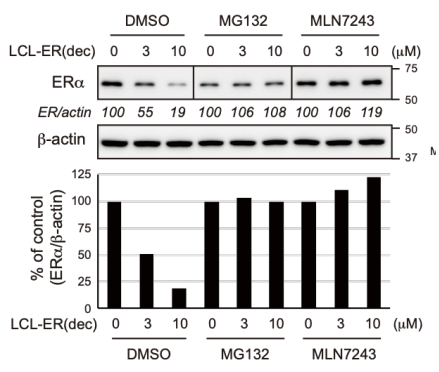

$\mathrm{F}$

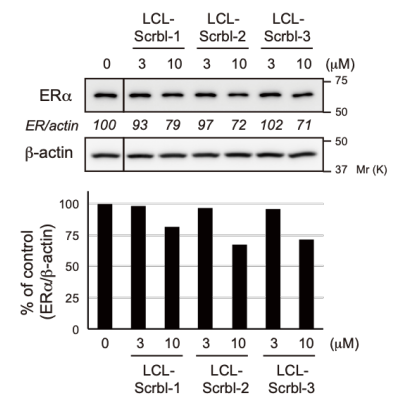

Figure 3. Degradation of the ER $\alpha$ via the UPS by the synthesized decoys. (A, B) The synthesized decoys selectively induced a reduction in the ER $\alpha$ protein level. MCF-7 cells were transiently transfected with the indicated concentrations of LCL-ER(dec), VH-ER(dec), or POM-ER(dec) for $24 \mathrm{~h}$. (C) The effect of UPS inhibitors on the LCL-ER(dec)-induced reduction of ER $\alpha$ levels. MCF-7 cells were transiently transfected with the indicated concentrations of LCL-ER(dec) in the presence or absence of $10 \mu \mathrm{M}$ MG132 or MLN7243 for $24 \mathrm{~h}$. (D-F) Degradation of the ER $\alpha$ protein by LCL-ER(dec) is IAP-ligand and DNAsequence dependent. MCF-7 cells were transiently transfected with the indicated concentrations of LCL-ER(dec), ER(dec), NMeLCL-ER(dec), LCL-Scramble-1, LCL-Scramble-2, or LCL-Scramble-3 for $24 \mathrm{~h}$. Whole-cell lysates were analyzed by western blotting with the indicated antibodies; representative data are shown. The numbers below the ER $\alpha$ panels represent the ER/actin ratios, normalized by designating the expression using the vehicle control (condition without a decoy) as 100\%. The changes in protein levels were reproducible between two independent experiments. The data in the bar graph are the mean of two results. Abbreviations; AR: androgen receptor, AhR: aryl hydrocarbon receptor, BRD4: bromodomain-containing protein 4, GAPDH: glyceraldehyde 3-phosphate dehydrogenase, CRABP2: cellular retinoic acid binding protein 2.

ER(dec), and $50.5 \mathrm{nM}$ for POM-ER(dec). These results indicated that the binding activity of the decoy ER(dec) to the ER $\alpha$ was not weakened by the linking of ER(dec) to the E3 ligase ligand, and the compounds had sufficient binding ability to the $\mathrm{ER} \alpha$ regardless of the type of the E3 ligase ligand (Table 1). The binding activities of LCL-Scrbl-1-3 to the ER $\alpha$ were considerably weaker than that of ER(dec) composed of the consensus sequence, but some non-specific binding was observed at high concentrations (Figures S8 and S9).

Table 1. ER $\alpha$ binding affinity $\left(\mathrm{IC}_{50}\right)$ of $\mathrm{ER}(\mathrm{dec}), \mathrm{LCL}-$ ER(dec), VH-ER(dec), POM-ER(dec), LCL-Scrbl-1, LCLScrbl-2, and LCL-Scrbl-3.

\begin{tabular}{lll}
\hline Entry & Compound & IC $_{\mathbf{5 0}}(\mathbf{n M})$ \\
\hline $\mathbf{1}$ & ER $(\mathrm{dec})$ & 67.0 \\
$\mathbf{2}$ & LCL-ER $(\mathrm{dec})$ & 37.9 \\
$\mathbf{3}$ & VH-ER $(\mathrm{dec})$ & 50.4 \\
$\mathbf{4}$ & POM-ER $(\mathrm{dec})$ & 39.3 \\
$\mathbf{5}$ & LCL-Scrbl-1 & $10.8 \times 10^{3}$
\end{tabular}

\begin{tabular}{lll}
$\mathbf{6}$ & LCL-Scrbl-2 & $3.28 \times 10^{3}$ \\
$\mathbf{7}$ & LCL-Scrbl-3 & $8.16 \times 10^{3}$ \\
\hline
\end{tabular}

To investigate whether LCL-ER(dec), VH-ER(dec), and POM-ER(dec) have degradation activity against the ER $\alpha$, the effect of these E3 ligand-decoy conjugates on the protein level of ER $\alpha$ was evaluated by western blotting analysis using MCF7 breast cancer cells. All the decoys reduced the ER $\alpha$ protein level after $24 \mathrm{~h}$ of transfection, and LCL-ER(dec) had the most effect compared with the other decoys (Figure 3A). To investigate the selectivity for the target protein, the effect of these decoys on the levels of different proteins was examined (Figure 3B). None of the decoys effectively reduced the levels of other transcription factors (AR, AhR, and NF- $\mathrm{KB}$ p65), transcriptionrelated factors (p300 and BRD4), or proteins not related to transcription (CRABP2, GAPDH, and $\beta$-actin), indicating that these decoys selectively reduced the protein level of ER $\alpha$.

We next investigated the mechanism of the ER $\alpha$ reduction by focusing on LCL-ER(dec), which showed the highest activity, as shown in Figure 3A. The LCL-ER(dec)-induced reduction of the ER $\alpha$ was abrogated by co-treatment with the 


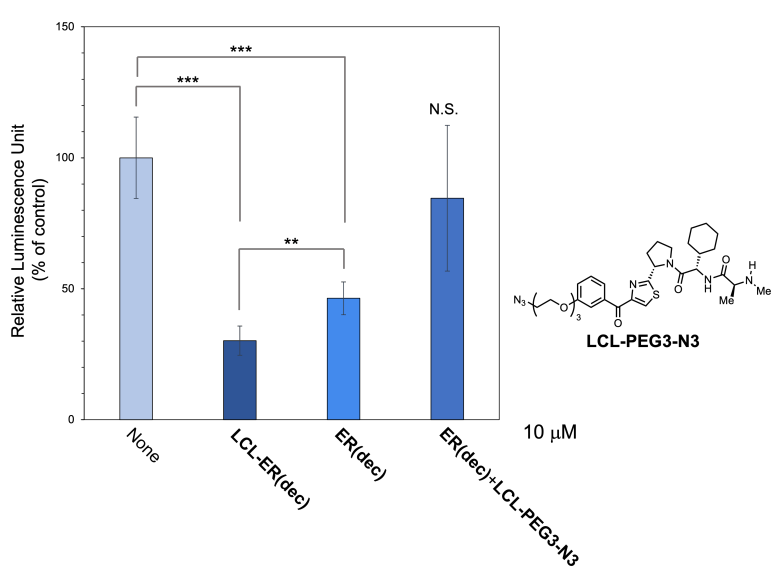

Figure 4. Inhibition of the estrogen-dependent transcriptional activity of ER $\alpha$ by LCL-ER(dec). MCF-7 cells were transiently transfected with a luciferase reporter plasmid containing three tandem copies of the ERE and control Renilla luciferase plasmid-SV40. After $24 \mathrm{~h}$, the cells were further transfected with $10 \mu \mathrm{M}$ concentrations of the indicated decoys in the presence of $0.3 \mathrm{nM} \beta$-estradiol for $24 \mathrm{~h}$. The ER $\alpha$-dependent transcriptional activity was evaluated by a luciferase assay, and the relative luciferase activity was normalized by designating the activity of the non-treated control (column 1) as $100 \%$. The data represent the mean \pm S.D. $(\mathrm{n}=4) . P$ values were determined using the unpaired

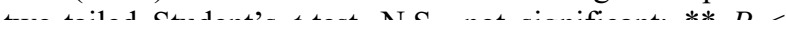
proteasome inhibitor MG132, and the ubiquitin-activating inhibitor MLN7243 (Figure 3C), suggesting that LCL-ER(dec) induced ubiquitin-proteasome system (UPS)-dependent degradation of the ER $\alpha$. Unlike LCL-ER(dec), ER(dec) did not induce degradation of the ER $\alpha$ protein (Figure 3D), indicating that conjugation of the LCL161 ligand is important for the degradation. In addition, the non-degradable control NMeLCLER(dec), which contains an $N$-methylated analog of the LCL161 ligand, did not induce ER $\alpha$ degradation (Figure 3E), suggesting that the ability to bind IAPs is critical for the degradation activity. To examine whether target recognition by the decoy ligands was DNA-sequence dependent, we synthesized LCL161 ligand-decoy chimeras using several different decoys composed of scrambled DNA sequences as the target ligands. These decoy chimeras did not show significant ER $\alpha$ degradation activity (Figure 3F), which correlated with their weak binding affinity to the ER $\alpha$ (Table 1). These results suggested that decoy oligonucleotides can be used in the development of chimeric molecules that induce DNA sequence-dependent degradation of target proteins.

Because the ER $\alpha$ plays an essential role in estrogen signaling, we examined the effect of LCL-ER(dec) on the estrogen-dependent transcriptional activity of the ER $\alpha$. In a luciferase assay using an ERE reporter, LCL-ER(dec) inhibited ER $\alpha$ dependent transcriptional activation by $\beta$-estradiol more effectively than ER(dec), which was in good agreement with the ER $\alpha$ degradation activity (Figure 4). A mixture of the decoy ligand ER(dec) and LCL-PEG3-N3 did not effectively decrease the transcription, indicating that the linking of the two ligands, ER(dec) and LCL-PEG3-N3, is crucial for effective inhibition. These results suggested that degradation of the ER $\alpha$ by LCL-ER(dec) leads to effective suppression of estrogen signaling.
In summary, we synthesized LCL-ER(dec) with the aim of developing a chimeric degrader using a decoy ligand that can be applied to a variety of TFs. For the target protein, we selected the ER $\alpha$, which has previously been shown to be degraded by chimeric molecules containing small molecules or peptides. The three chimeric molecules LCL-ER(dec), VH-ER(dec), and POM-ER(dec) were synthesized by conjugating the ER $\alpha$ decoy ER(dec) with LCL161, VH032, and POM, E3 ligase ligands commonly used in PROTACs and SNIPERs. Among these three chimeric molecules, LCL-ER(dec) showed the highest ER $\alpha$ degradation-inducing activity as assessed by western blotting. In contrast, the ER $\alpha$-non-binding chimeric molecules, LCL-Scrbl-1-3, did not reduce the protein levels of ER $\alpha$, suggesting that LCL-ER(dec) binds to the ER $\alpha$ in a sequence-specific manner to induce the degradation. In addition, the non-degradable control, NMeLCL-ER(dec), did not induce ER $\alpha$ degradation, suggesting that the ability to bind IAPs is critical for the degradation activity. Studies using UPS inhibitors suggested that LCL-ER(dec) degraded the ER $\alpha$ via the UPS. Recently, TF-PROTACs ${ }^{16}\left(\mathrm{O}^{\prime}\right.$ PROTACs $\left.{ }^{17}\right)$ targeting TFs using doublestranded decoy ligands have been reported, indicating that decoy-type PROTACs are also useful as a new type of POI inducer. In the future, we aim to apply the methods developed in this study to the design of degradation inducers for transcription-related factors that are difficult to target.

\section{ASSOCIATED CONTENT}

\section{Supporting Information}

The Supporting Information is available free of charge on the ACS Publications website at DOI:

The synthetic procedures for all compounds listed in this manuscript and the protocols for the in vitro assays (binding and protein degradation assays) (PDF).

\section{AUTHOR INFORMATION}

\section{Corresponding Author}

* Tel: +81-44-270-6578, Fax: +81-44-270-6578, E-mail: demizu@nihs.go.jp (Y. Demizu). Tel: +81-44-270-6537, E-mail: n-ohoka@nihs.go.jp (N. Ohoka).

\section{Author Contributions}

Miyako Naganuma, N.O., G.T., and H.T. performed the experiments and analyzed results. Miyako Naganuma, N.O., T.I., Mikihiko Naito, and Y.D. designed the research and wrote the paper. All authors discussed the results and commented on the manuscript.

\section{ORCID}

Miyako Naganuma : 0000-0003-0011-6268

Nobumichi Ohoka : 0000-0002-0533-0610

Takao Inoue : 0000-0002-6507-9480

Mikihiko Naito : 0000-0003-0451-1337

Yosuke Demizu : 0000-0001-7521-4861

\section{Funding Sources}

This study was supported in part by grants from AMED under grant numbers JP21mk0101197 (to Y.D. and N.O.) and JP21ak0101073 (to N.O., T.I. and Y.D.), $21 \mathrm{mk} 0101187$ (to N.O. and T.I.) and JP21am0401003 (to T.I.), the Japan Society for the Promotion of 
Science (KAKENHI, grants JP21K05320 to Y.D.; JP18H05502 to Mikihiko Naito and Y.D.; JP18K06567 and JP21K06490 to N.O.; JSPS Fellows 2121J23036 to Miyako Naganuma), Terumo Foundation for Life Sciences and Arts (to Y.D.), Takeda Science Foundation (to Y.D.), the Naito Foundation (to Y.D.), the Sumitomo Foundation (to Y.D.), the Novartis Foundation (Japan) for the Promotion of Science (to Y.D.), Japan Foundation of Applied Enzymology (to Y.D.), Kobayashi Foundation for Cancer Research (to Y.D.) and Foundation for Promotion of Cancer Research in Japan (to Y.D.)

\section{ACKNOWLEDGMENT}

We thank Edanz (https://www.jp.edanz.com/ac) for editing a draft of this manuscript.

\section{ABBREVIATIONS}

PROTACs, proteolysis targeting chimeras; IAP, inhibitor of apoptosis protein; SNIPERs, specific and non-genetic IAPdependent protein erasers; UPS, ubiquitin proteasome system; TF, transcription factor; ER $\alpha$, estrogen receptor $\alpha$; POI, protein of interest; VHL, von Hippel-Lindau protein; NOTCH1, neurogenic locus notch homolog protein 1; 4-OHT, 4-hydroxytamoxifen; ERE, estrogen-responsive element; FAM, fluorescein; $\mathrm{CD}$, circular dichroism; $T_{\mathrm{m}}$, melting temperature.

\section{REFERENCES}

(1) Ferguson, F. M.; Gray, N. S., Kinase inhibitors: the road ahead. Nat. Rev. Drug Discov. 2018, 17 (5), 353-376.

(2) Nelson, A. L.; Dhimolea, E.; Reichert, J. M., Development trends for human monoclonal antibody therapeutics. Nat. Rev Drug Discov. 2010, 9 (10), 767-774.

(3) Burslem, G. M.; Crews, C. M., Small-Molecule Modulation of Protein Homeostasis. Chem. Rev. 2017, 117 (17), 1126911301.

(4) Itoh, Y.; Ishikawa, M.; Naito, M.; Hashimoto, Y., Protein Knockdown Using Methyl Bestatin-Ligand Hybrid Molecules: Design and Synthesis of Inducers of UbiquitinationMediated Degradation of Cellular Retinoic Acid-Binding Proteins. J. Am. Chem. Soc. 2010, 132 (16), 5820-5826.

(5) Yokoo, H.; Ohoka, N.; Naito, M.; Demizu, Y., Design and synthesis of peptide-based chimeric molecules to induce degradation of the estrogen and androgen receptors. Bioorg. Med. Chem. 2020, 28 (15), 115595.

(6) Ohoka, N.; Misawa, T.; Kurihara, M.; Demizu, Y.; Naito, M., Development of a peptide-based inducer of protein degradation targeting NOTCH1. Bioorg. Med. Chem. Lett. 2017, 27 (22), 4985-4988.

(7) Keefe, A. D.; Pai, S.; Ellington, A., Aptamers as therapeutics. Nat. Rev. Drug Discov. 2010, 9 (7), 537-550.
(8) Morishita, R.; Higaki, J.; Tomita, N.; Ogihara, T., Application of transcription factor "decoy" strategy as means of gene therapy and study of gene expression in cardiovascular disease. Circ. Res. 1998, 82 (10), 1023-1028.

(9) Pawlak, M.; Lefebvre, P.; Staels, B., General Molecular Biology and Architecture of Nuclear Receptors. Curr. Top. Med. Chem. 2012, 12 (6), 486-504.

(10) Kumar, V.; Chambon, P., THE ESTROGEN-RECEPTOR BINDS TIGHTLY TO ITS RESPONSIVE ELEMENT AS A LIGAND-INDUCED HOMODIMER. Cell 1988, 55 (1), 145-156.

(11) Ohoka, N.; Okuhira, K.; Ito, M.; Nagai, K.; Shibata, N.; Hattori, T.; Ujikawa, O.; Shimokawa, K.; Sano, O.; Koyama, R.; Fujita, H.; Teratani, M.; Matsumoto, H.; Imaeda, Y.; Nara, H.; Cho, N.; Naito, M., In Vivo Knockdown of Pathogenic Proteins via Specific and Nongenetic Inhibitor of Apoptosis Protein (IAP)-dependent Protein Erasers (SNIPERs). J. Biol. Chem. 2017, 292 (11), 4556-4570.

(12) Ohoka, N.; Morita, Y.; Nagai, K.; Shimokawa, K.; Ujikawa, O.; Fujimori, I.; Ito, M.; Hayase, Y.; Okuhira, K.; Shibata, N.; Hattori, T.; Sameshima, T.; Sano, O.; Koyama, R.; Imaeda, Y.; Nara, H.; Cho, N.; Naito, M., Derivatization of inhibitor of apoptosis protein (IAP) ligands yields improved inducers of estrogen receptor $\alpha$ degradation. J. Biol. Chem. 2018, 293 (18), 6776-6790.

(13) Kleinhitpass, L.; Schorpp, M.; Wagner, U.; Ryffel, G. U., AN ESTROGEN-RESPONSIVE ELEMENT DERIVED FROM THE 5' FLANKING REGION OF THE XENOPUS VITELLOGENIN A2 GENE FUNCTIONS IN TRANSFECTED HUMAN-CELLS. Cell 1986, 46 (7), 1053 1061.

(14) Schwabe, J. W. R.; Chapman, L.; Finch, J. T.; Rhodes, D., THE CRYSTAL-STRUCTURE OF THE ESTROGENRECEPTOR DNA-BINDING DOMAIN BOUND TO DNA - HOW RECEPTORS DISCRIMINATE BETWEEN THEIR RESPONSE ELEMENTS. Cell 1993, 75 (3), 567-578.

(15) Zengerle, M.; Chan, K. H.; Ciulli, A., Selective Small Molecule Induced Degradation of the BET Bromodomain Protein BRD4. ACS Chem. Biol. 2015, 10 (8), 1770-1777.

(16) Zhang, F. Q.; Wu, Z. W.; Chen, P.; Zhang, J.; Wang, T.; Zhou, J. P.; Zhang, H. B., Discovery of a new class of PROTAC BRD4 degraders based on a dihydroquinazolinone derivative and lenalidomide/pomalidomide. Bioorg. Med. Chem. 2020 , $28(1)$.

(17) Liu, J.; Chen, H.; Kaniskan, H. U.; Xie, L.; Chen, X.; Jin, J.; Wei, W. Y., TF-PROTACs Enable Targeted Degradation of Transcription Factors. J. Am. Chem. Soc. 2021, 143 (23), 8902-8910.

(18) Shao, J.; Yan, Y.; Ding, D.; Wang, D.; He, Y.; Pan, Y.; Yan, W.; Kharbanda, A.; Li, H. Y.; Huang, H., Destruction of DNA-Binding Proteins by Programmable Oligonucleotide PROTAC (O'PROTAC): Effective Targeting of LEF1 and ERG. $A d v$. Sci. 2021, e2102555. 


\section{Development of chimeric molecules that degrade the estrogen receptor using decoy oligonucleotide ligands}

Miyako Naganuma, Nobumichi Ohoka, Genichiro Tsuji, Haruna Tsujimura, Kenji Matsuno, Takao Inoue, Mikihiko Naito, and Yosuke Demizu

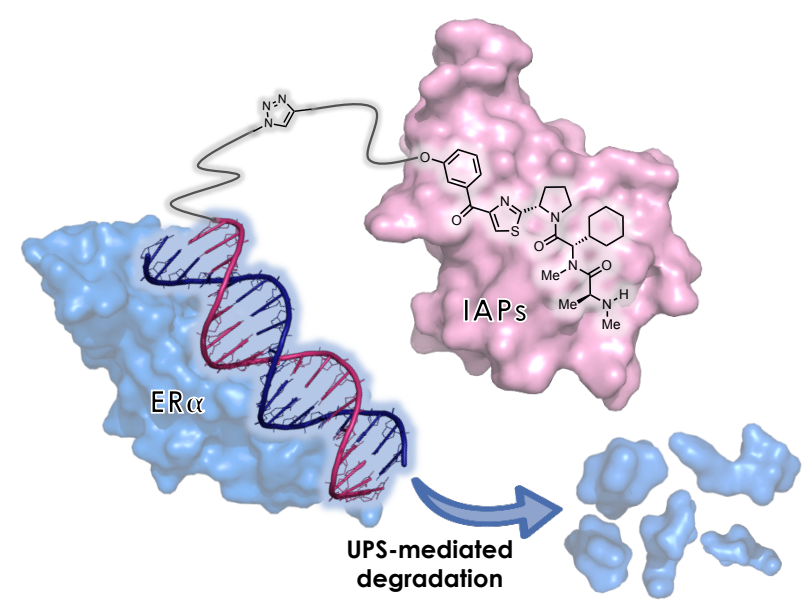


Protein degradation technology using hybrid small molecules (known as PROTACs and SNIPERs) is an emerging drug development strategy for use in drug discovery and biological research. In this study, a decoy ligand-based estrogen receptor $\alpha$ $(\mathrm{ER} \alpha)$ degrader LCL-ER( $\mathrm{dec})$ was designed and synthesized and the binding affinity and degradation activity toward the ER $\alpha$ were evaluated. LCL-ER(dec) was revealed to selectively degrade the ER $\alpha$ via the ubiquitin-proteasome system. Because we were able to show that decoys were able to be used as the ligands for protein degraders, we expect that this concept will lead to the expansion of the number of proteins that can be targeted. 